\title{
Asymptotically Optimal Channel Feedback Protocol Design for Cognitive Multiple Access Channels
}

\author{
Ehsan Nekouei \\ Department of Electrical and Electronic \\ Engineering, The University of \\ Melbourne, VIC, Australia
}

\author{
Hazer Inaltekin \\ Department of Electrical and Electron
Engineering, Antalya International \\ University, Antalya, Turkey
}

\author{
Subhrakanti Dey \\ Department of Electrical and Electronic \\ Engineering, The University of \\ Melbourne, VIC, Australia
}

\begin{abstract}
In cognitive multiple access networks, primarysecondary feedback links are needed to convey secondary transmitter primary base station (STPB) channel gains from the primary base station (PBS) to the secondary base station (SBS). To reduce the amount of feedback exchange between PBS and SBS, this paper proposes a feedback control protocol called $K$ smallest channel gains ( $K$-SCG) feedback protocol in which the PBS feeds back the $K_{N}$ smallest STPB channel gains, out of $N$ of them, to the SBS. We study the performance of $K$-SCG feedback protocol for total power and interference limited (TPIL) networks when transmit powers of secondary users (SUs) are optimally allocated. In TPIL networks, transmit powers of SUs are limited by an average total power constraint as well as a constraint on the average total interference power that they cause to the PBS. It is shown that for $K_{N}=N^{\delta}$ with $\delta \in(0,1), K$ SCG feedback protocol is asymptotically optimal, i.e., secondary network throughput under $K$-SCG and full feedback protocols scales according to $\frac{1}{n_{h}} \log \log (N)$ where $n_{h}$ is a parameter obtained from the distribution of secondary transmitter secondary base station (STSB) channel power gains, and $N$ is the number of SUs. It is also shown that for $K_{N}=o(N)$, the interference power at the PBS converges to zero almost surely and in mean as $N$ becomes large. This result implies that for $N$ large enough, the secondary network just requires the indices of SUs corresponding to the $K_{N}$ smallest STPB channel gains for performing jointly optimal user scheduling and power allocation rather than the actual realizations of STPB channel gains.
\end{abstract}

\section{INTRODUCTION}

Cognitive radio technology alleviates the spectrum scarcity problem by allowing cognitive users (also called secondary users or SUs) to share the same bandwidth with legacy users (also called primary users or PUs) provided that certain level of quality-of-service (QoS) delivered to PUs. Various paradigms for the coexistence of primary and secondary networks have been proposed such as underlay, overlay and interweave paradigms [1]. The paradigm of interest in this paper is the underlay paradigm, which is also known as spectrum sharing technique [2]. Under the spectrum sharing setup, SUs transmit simultaneously with PUs, but they are required to manage their interference at the primary receiver in order to guarantee a predefined level of QoS at the primary network.

In cognitive multiple access networks, optimal resource utilization and interference management tasks rely heavily on the

This research was supported in part by the European Union Research Executive Agency Marie Curie FP7-Reintegration-Grants under Grant PCIG10GA-2011-303713 and in part by the Australian Research Council under Grant DP-11-0102729. knowledge of STPB channel gains that are conveyed from the PBS to the SBS by means of feedback links or a band manager mediating communication between primary and secondary networks [2], [3] (see also Fig. 1). For large numbers of SUs, it becomes impractical for the PBS to convey all STSB channel gains to the SBS due to various physical restrictions such as feedback link capacity limitations and energy constraints. This paper studies the effect of feedback link limitations on the throughput scaling behavior of secondary networks.

Throughput scaling behavior of cognitive radio networks has been studied in some recent papers. The authors in [4] obtained the capacity scaling laws for a cognitive multiple access channel (CMAC) under peak transmit power and peak interference power constraints. In [5], Zhang et al. extended these results to cognitive broadcast channels and cognitive parallel access channels. In [6], Nekouei et al. established logarithmic and double-logarithmic capacity scaling behavior for interference-limited and power-interference-limited CMACs, respectively, when transmit powers of SUs are optimally allocated. In [7], the double-logarithmic capacity scaling laws were established for a secondary network with $N$ secondary transmitter-receiver pairs sharing $M$ frequency bands with a primary network under optimum pairing of SUs and frequency bands. In [8], multiuser and multispectrum diversity gains were studied for a cognitive broadcast network sharing multiple orthogonal frequency bands with a primary network.

In this paper, we consider a secondary network with $N$ SUs under an average total power constraint and an average interference power constraint as shown in Fig 1. Different from previous works, we consider a more realistic scenario in which the PBS is able to send the STPB channel gains of at most $K_{N}$ SUs to the SBS, where $K_{N}$ is a function of $N$. To protect the primary transmission, the PBS picks the least harmful SUs by feeding back the channel gain $g_{i}$ of the SU- $i$ if and only if $g_{i} \leq g_{K_{N}: N}$, where $g_{K_{N}: N}$ is the $K_{N}$ th smallest value in the set $\left\{g_{i}\right\}_{i=1}^{N}$. We refer to this feedback policy as the $K$-smallest channel gain ( $K$-SCG) feedback protocol. $K_{N}=N$ case is named as the full-feedback protocol. To avoid any harmful interference at the PBS, the SBS schedules an SU if its STPB channel gain is available at the SBS. We study throughput scaling behavior of secondary networks under both $K$-SCG and full-feedback protocols when transmit powers of SUs are optimally allocated, and distributions of STSB and STPB 
channel gains belong to a fairly large class of distribution functions called class $\mathcal{C}$ distributions ( see Definition 1).

Our results indicate that the secondary network throughput under the $K$-SCG and full-feedback protocols scales according to $\frac{1}{n_{h}} \log \log \left(K_{N}\right)$ and $\frac{1}{n_{h}} \log \log (N)$, respectively. $n_{h}$ is a parameter determined from the distribution of STSB channel power gains. Hence, for $K_{N}=N^{\delta}$ with $0<\delta<1$, the secondary network can achieve the same throughput scaling as with the full-feedback case. For $K_{N}$ growing to infinity as $K_{N}=o(N)$, we show that the worst-case interference power at the PBS converges to zero almost surely (a.s.) and also in mean as $N$ tends to infinity. This result implies that the interference constraint cannot be satisfied with equality for $N$ large enough. Hence, we can relax the interference constraint, and the SBS just requires the indices of the SUs for which $g_{i} \leq g_{K_{N}: N}$ rather than the actual realizations of the STPB channel gains, which further reduces the amount of feedback required between two networks.

The rest of the paper is organized as follows. Section II describes the system model and network configuration along with our modeling assumptions. Section III derives and presents the secondary network throughput scaling laws under $K$-SCG and full feedback policies. Section IV presents some numerical results to illustrate the derived scaling laws. Section $\mathrm{V}$ concludes the paper.

\section{SySTEM MOdel}

We consider a cognitive multiple access network where $N$ SUs transmit data to an SBS and interfere with the signal reception at a PBS as shown Fig. 1. Let $h_{i}$ and $g_{i}$ represent the fading power gains for the $i$ th STSB and STPB links, respectively. We assume that $\left\{h_{i}\right\}_{i=1}^{N}$ and $\left\{g_{i}\right\}_{i=1}^{N}$ are collections of independent and identically distributed (i.i.d.) random variables. The random vectors $\boldsymbol{h}=\left[h_{1}, h_{2}, \ldots, h_{N}\right]^{\top}$ and $\boldsymbol{g}=\left[g_{1}, g_{2}, \ldots, g_{N}\right]^{\top}$ are also independent. The fading model is the classical ergodic block fading model.

Definition 1: We say that the cumulative distribution function $(\mathrm{CDF})$ of a random variable $X$, i.e., $F(x)$, belongs to the class $\mathcal{C}$ distributions if it satisfies the following properties:

- $F(x)$ has a positive support, i.e., $F(x)=0$ for $x \leq 0$.

- $F(x)$ is continuous and strictly increasing for $x>0$.

- The tail of $F(x)$ decays double exponentially, i.e., there exist constants $\alpha>0, \beta>0, n>0, l \in \mathbb{R}$ and a function $H(x)$ satisfying $H(x)=o\left(x^{n}\right)$ such that $\lim _{x \rightarrow \infty} \frac{1-F(x)}{\alpha x^{l} e^{\left(-\beta x^{n}+H(x)\right)}}=1$.

- $F(x)$ varies regularly near the origin, i.e., there exist constants $\eta>0$ and $\gamma>0$ such that $\lim _{x \downarrow 0} \frac{F(x)}{\eta x^{\gamma}}=1$.

In this paper, we assume that the CDFs of all fading power gains belong to the class $\mathcal{C}$ distributions. The parameters characterizing the behavior of the distribution of fading power gains around zero and infinity are illustrated in Table I for the commonly used fading models in the literature. To avoid any confusion, we represent these parameters with subscript $h$ for

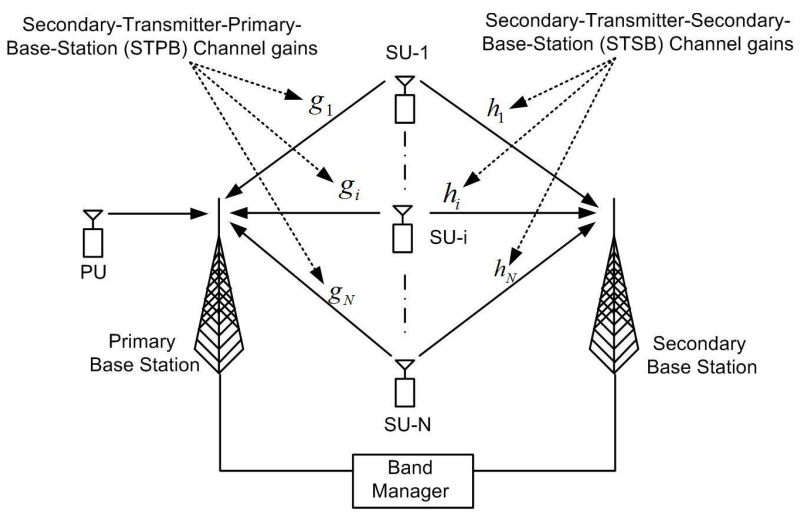

Fig. 1. $\quad N$ SUs forming a multiple access channel to the SBS and interfering with signal reception at the PBS.

STSB channel gains and with subscript $g$ for STPB channel gains, e.g., $\eta_{g}$ or $\eta_{h}$.

We define a power allocation policy $\boldsymbol{P}(\boldsymbol{h}, \boldsymbol{g})=$ $\left[P_{1}(\boldsymbol{h}, \boldsymbol{g}), \cdots, P_{N}(\boldsymbol{h}, \boldsymbol{g})\right]^{\top}$ as a mapping from $\mathbb{R}_{+}^{2 N}$ to $\mathbb{R}_{+}^{N}$ in which $P_{i}(\boldsymbol{h}, \boldsymbol{g})$ is the transmit power of SU- $i$. We study the throughput scaling of secondary networks under the following constraints:

$$
\left\{\begin{array}{l}
\mathrm{E}_{\boldsymbol{h}, \boldsymbol{g}}\left[\mathbf{1}^{\top} \boldsymbol{P}(\boldsymbol{h}, \boldsymbol{g})\right] \leq P_{\mathrm{ave}}, \\
\mathrm{E}_{\boldsymbol{h}, \boldsymbol{g}}\left[\boldsymbol{g}^{\top} \boldsymbol{P}(\boldsymbol{h}, \boldsymbol{g})\right] \leq Q_{\mathrm{ave}}, \\
P_{i}(\boldsymbol{h}, \boldsymbol{g}) 1_{\left\{g_{i}>g_{K_{N}: N}\right\}}=0 \quad 1 \leq i \leq N,
\end{array}\right.
$$

where (1a) and (1b) are average total power and average total interference power constrains, respectively. (1c) is a constraint to guarantee that a $\mathrm{SU}$ is allowed to transmit only if its STPB channel gain is available at the SBS. Note that such average total power constraints have been employed to study the capacity of multiple access channels before (e.g., see [6] and references therein), but only for very specific channel gain distributions under the full-feedback case. We examine the secondary network throughput scaling behavior in two communication scenarios (CoSs): $\operatorname{CoS}_{\mathrm{TPIL}}^{\mathrm{F}}$ and $\operatorname{CoS}_{\mathrm{TPIL}}^{K}$. In $\mathrm{CoS}_{\mathrm{TPIL}}^{\mathrm{F}}$, transmit powers of SUs are limited by an average total power constraint and an average total interference power constraint. In this CoS, the PBS feeds back all STSB channel gains to the SBS. Thus, transmit powers of SUs are allocated according to the solution of the following optimization problem:

$$
\begin{aligned}
& \max _{\boldsymbol{P}(\boldsymbol{h}, \boldsymbol{g})} \mathrm{E}_{\boldsymbol{h}, \boldsymbol{g}}\left[\log \left(1+\boldsymbol{h}^{\top} \boldsymbol{P}(\boldsymbol{h}, \boldsymbol{g})\right)\right] . \\
& \text { subject to : (1a) and (1b) }
\end{aligned}
$$

The solution to (2) was derived in [6], [9] as

$$
\begin{aligned}
& P_{i, N}^{\star}(\boldsymbol{h}, \boldsymbol{g}) \\
& =\left\{\begin{array}{cc}
\left(\frac{1}{\lambda_{N}+\mu_{N} g_{i}}-\frac{1}{h_{i}}\right)^{+} & \text {if } i=\underset{\text { otherwise }}{\arg \max _{1 \leq j \leq N} \frac{h_{j}}{\lambda_{N}+\mu_{N} g_{j}}} .
\end{array}\right.
\end{aligned}
$$

Let $R_{\mathrm{TPIL}}^{\mathrm{F}}(N)$ be the sum-rate of the secondary network in $\operatorname{CoS}_{\mathrm{TPIL}}^{\mathrm{F}}$. Then, it follows directly that

$$
R_{\mathrm{TPIL}}^{\mathrm{F}}(N)=\mathrm{E}\left[\log \left(X_{N}^{\star}\left(\lambda_{N}, \mu_{N}\right)\right) 1_{\left\{X_{N}^{\star}\left(\lambda_{N}, \mu_{N}\right) \geq 1\right\}}\right],
$$


TABLE I

COMMON FADING CHANNEL MODELS AND THEIR PARAMETERS

\begin{tabular}{cccccccc}
\hline \multirow{2}{*}{ Channel Model } & $\alpha$ & $l$ & $\beta$ & $n$ & $h(x)$ & $\eta$ & $\gamma$ \\
\cline { 2 - 9 } & 1 & 0 & 1 & 1 & 0 & 1 & 1 \\
\hline Rayleigh & $\frac{1}{\sqrt{7}}$ & $-\frac{1}{4}$ & $K_{f}+1$ & 1 & $2 \sqrt{K_{f}\left(K_{f}+1\right) x}$ & $\frac{K_{f}+1}{\mathrm{e}^{K_{f}}}$ & 1 \\
\hline Rician & $\frac{m^{m-1}}{\Gamma(m)}$ & $m-1$ & $m$ & 1 & 0 & $\frac{m^{m-1}}{\Gamma(m)}$ & $m$ \\
\hline Nakagami- $m$ & 1 & 0 & $\Gamma^{\frac{c}{2}}\left(1+\frac{2}{c}\right)$ & $\frac{c}{2}$ & 0 & $\Gamma^{\frac{c}{2}}\left(1+\frac{2}{c}\right)$ & $\frac{c}{2}$ \\
\hline Weibull & & & & & & &
\end{tabular}

where $\lambda_{N}$ and $\mu_{N}$ are Lagrange multipliers associated with the average total transmit and interference power constraints, respectively, and $X_{N}^{\star}\left(\lambda_{N}, \mu_{N}\right)=\max _{1 \leq i \leq N} \frac{h_{i}}{\lambda_{N}+\mu_{N} g_{i}}$.

On the other hand, transmit powers of SUs in $\operatorname{CoS}_{\text {TPIL }}^{K}$ are also limited by the extra feedback constraint in (1c), besides the average total transmit and interference power constraints above. Hence, transmit powers of SUs in this case are allocated according to the solution of the following optimization problem:

$$
\begin{aligned}
& \max _{\boldsymbol{P}(\boldsymbol{h}, \boldsymbol{g})} \mathrm{E}_{\boldsymbol{h}, \boldsymbol{g}}\left[\log \left(1+\boldsymbol{h}^{\top} \boldsymbol{P}(\boldsymbol{h}, \boldsymbol{g})\right)\right] . \\
& \text { subject to : (1a), (1b), (1c) }
\end{aligned}
$$

Lemma 1: Let's define $\pi(j)$ as mapping from $\left\{1, \cdots, K_{N}\right\}$ to $\{1, \cdots, N\}$ such that

$$
\pi(j)=i \quad \text { if } \quad g_{i}=g_{j: N} .
$$

Then, the solution for (3) is given by

$$
=\left\{\begin{array}{c}
P_{i, K_{N}}^{\star}\left(\frac{1}{\lambda_{N}+\mu_{N} g_{i}}-\frac{1}{h_{i}}\right)^{+} \\
0
\end{array} \text { if } i=\pi\left(\arg \max _{\substack{1 \leq j \leq K_{N} \\
\text { otherwise }}} \frac{h_{\pi(j)}}{\lambda_{N}+\mu_{N} g_{\pi(j)}}\right)\right.
$$

Proof: Follows directly by inspecting the structure of the solution given for (2).

Hence, the sum-rate in $\operatorname{CoS}_{\mathrm{TPIL}}^{K}$ scenario is given by

$$
R_{\mathrm{TPIL}}^{K}\left(K_{N}\right)=\mathrm{E}\left[\log \left(X_{K_{N}}^{\star}\left(\lambda_{N}, \mu_{N}\right)\right) 1_{\left\{X_{K_{N}}^{\star}\left(\lambda_{N}, \mu_{N}\right) \geq 1\right\}}\right],
$$

where $X_{K_{N}}^{\star}\left(\lambda_{N}, \mu_{N}\right)=\max _{1 \leq j \leq K_{N}} \frac{h_{\pi(j)}}{\lambda_{N}+\mu_{N} g_{\pi(j)}}$.

\section{RESULTS AND Discussions}

In this section, we study the throughput scaling behavior of TPIL networks under $\operatorname{CoS}_{\text {TPIL }}^{\mathrm{F}}$ and $\mathrm{CoS}_{\mathrm{TPIL}}^{K}$. The next theorem establishes the scaling behavior for $R_{\mathrm{TPIL}}^{\mathrm{F}}(N)$ and $R_{\mathrm{TPIL}}^{K}\left(K_{N}\right)$.

Theorem 1: Let $K_{N}$ grow to infinity at a rate $K_{N}=o(N)$. Then,

$$
\lim _{N \rightarrow \infty} \frac{R_{\mathrm{TPIL}}^{K}\left(K_{N}\right)}{\log \left(\log \left(K_{N}\right)\right)}=\lim _{N \rightarrow \infty} \frac{R_{\mathrm{TPIL}}^{\mathrm{F}}(N)}{\log (\log (N))}=\frac{1}{n_{h}} .
$$

Proof: Please see Appendix A.

Theorem 1 implies that for $K_{N}=N^{\delta}$ with $\delta \in(0,1)$, the secondary network throughput scaling behavior under $\mathrm{CoS}_{\mathrm{TPIL}}^{\mathrm{F}}$ and $\mathrm{CoS}_{\mathrm{TPIL}}^{K}$ are the same. Hence, under the $K$ SCG feedback protocol, the amount of feedback between the PBS and SBS can be dramatically reduced while the secondary network still achieves the same scaling behavior as the one achieved by the full-feedback protocol. Moreover, Theorem 1 reveals that secondary network scaling behavior under $\operatorname{CoS}_{\mathrm{TPIL}}^{\mathrm{F}}$ and $\operatorname{CoS}_{\mathrm{TPIL}}^{K}$ is controlled by a pre-log factor of $\frac{1}{n_{h}}$. The pre-log factor is equal to 1 for Rayleigh, Rician and Nakagami- $m$ distributed STSB channel gains, and to $\frac{2}{c}$ for the Weibull distributed STSB channel gains where $c$ is the Weibull fading parameter. Based on our analysis in Appendix A, we can characterize the effect of $P_{\text {ave }}$ and other second order effects of fading parameters on the secondary network throughput under $\mathrm{CoS}_{\mathrm{TPIL}}^{\mathrm{F}}$ for finite number of SUs. That is, $R_{\mathrm{TPIL}}^{\mathrm{F}}(N)$ can be upper and lower bounded as (4) for all $\epsilon>0$ and $N$ large enough (see (14)). Based on (4), we conclude that the average power constraint $P_{\text {ave }}$ has a logarithmic effect on the secondary network throughput under $\mathrm{CoS}_{\mathrm{TPIL}}^{\mathrm{F}}$, and the second order effect of fading parameters on $R_{\mathrm{TPIL}}^{\mathrm{F}}(N)$ can be expressed as $\frac{1}{n_{h}} \log \left(\frac{1}{\beta_{h}}\right)$. Moreover, we note that $P_{\text {ave }}$ and $\frac{1}{n_{h}} \log \left(\frac{1}{\beta_{h}}\right)$ have similar effects on the secondary network throughput under $\operatorname{CoS}_{\text {TPIL }}^{K}$ (see (12)).

The next theorem establishes an important convergence behavior of interference at the PBS under $\operatorname{CoS}_{\mathrm{TPIL}}^{K}$ as the number of SUs grows large.

Theorem 2: Let $\mathcal{I}_{K_{N}}$ be the secondary network interference power at the PBS under $\operatorname{CoS}_{\text {TPIL }}^{K}$. For $K_{N}=o(N)$, $\lim _{N \rightarrow \infty} \mathcal{I}_{K_{N}}=0$ a.s. and $\lim _{N \rightarrow \infty} \mathrm{E}\left[\mathcal{I}_{K_{N}}\right]=0$.

Proof: Please see Appendix B.

Theorem 2 implies that the average interference power constraint at the PBS cannot be satisfied with equality for $N$ large enough. Therefore, Lagrange multiplies associated with the average interference constraint are set to zero for all $N$ large enough. Hence, the SBS just requires the index set $I_{K_{N}}=\left\{i: i=\pi(j), 1 \leq j \leq K_{N}\right\}$ for scheduling and optimal power allocation. This provides an extra reduction in the total primary-secondary feedback load. 


$$
\begin{aligned}
\frac{(1-\epsilon)}{n_{h}} \log \log (N)+\log \left(P_{\text {ave }}\right)+\frac{1}{n_{h}} \log \left(\frac{1}{\beta_{h}}\right)+O & (1) \leq R_{\mathrm{TPIL}}^{\mathrm{F}}(N) \leq \\
& \frac{(1+\epsilon)}{n_{h}} \log \log (N)+\log \left(P_{\text {ave }}\right)+\frac{1}{n_{h}} \log \left(\frac{1}{\beta_{h}}\right)+O(1)
\end{aligned}
$$

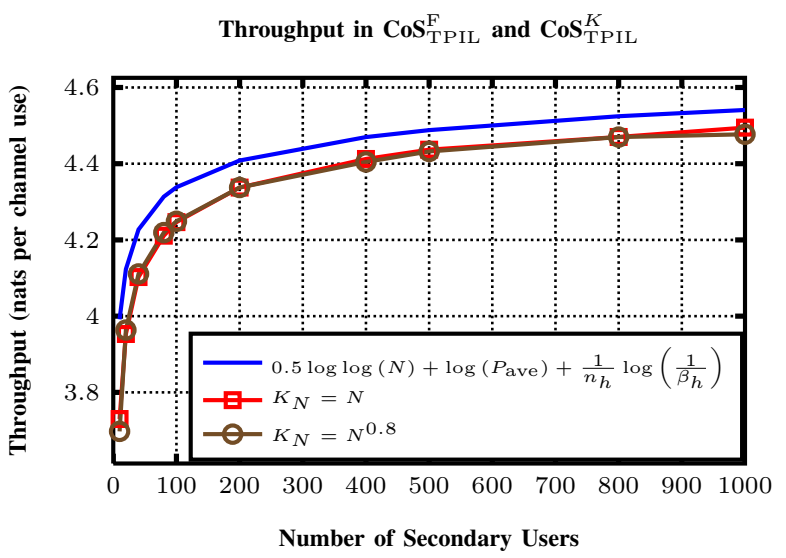

Fig. 2. Secondary network throughput scaling under $\operatorname{CoS}_{\mathrm{TPIL}}^{\mathrm{F}}$ and $\operatorname{CoS}_{\mathrm{TPIL}}^{K}$. $P_{\text {ave }}$ and $Q_{\text {ave }}$ are set to $15 \mathrm{~dB}$ and $0 \mathrm{~dB}$, respectively. STSB and STPB channel gains are distributed according to Weibull and Nakagami- $m$ fading models, respectively, with $c=4$ and $m=0.5$.

\section{Simulation Results}

In this section, we present our simulation results for secondary network throughput under $K$-SCG and full feedback protocols. In our simulations, $P_{\text {ave }}$ and $Q_{\text {ave }}$ are set to $15 \mathrm{~dB}$ and $0 \mathrm{~dB}$, respectively.

Figures 2 and 3 demonstrate throughput scaling behavior of the secondary network under $\operatorname{CoS}_{\mathrm{TPIL}}^{\mathrm{F}}$ and $\mathrm{COS}_{\mathrm{TPIL}}^{K}$ as a function of the number of SUs. In Figs. 2 and 3, the curves with $K_{N}=N$ represent the secondary network throughput under $\operatorname{CoS}_{\text {TPIL }}^{\mathrm{F}}$, and the curves with $K_{N}=N^{0.8}$ represent the secondary network throughput under $\operatorname{CoS}_{\mathrm{TPIL}}^{K}$. In both figures, STSB and STPB channel gains are distributed according to Weibull and Nakagami- $m$ fading models, respectively. Weibull fading parameter, $c$, is set to 4 in Fig. 1 and to 1 in Fig. 2. In both figures, Nakagami fading parameter $m$ is set to 0.5 . In both figures, the throughput of secondary network under $\mathrm{CoS}_{\mathrm{TPIL}}^{\mathrm{F}}$ and $\mathrm{CoS}_{\mathrm{TPIL}}^{K}$ scales according to $\frac{2}{c} \log \log (N)$, as predicted by Theorem 1. In particular, the closeness of simulated data rates and $\frac{1}{n_{h}} \log \log (N)+\log \left(P_{\text {ave }}\right)+\frac{1}{n_{h}} \log \left(\frac{1}{\beta_{h}}\right)$ curves further indicates the logarithmic effect of $P_{\text {ave }}$ as well as the other second order effects on the secondary network throughput under $\operatorname{CoS}_{\mathrm{TPIL}}^{\mathrm{F}}$ and $\operatorname{CoS}_{\mathrm{TPIL}}^{K}$. Finally, as Figs. 2 and 3 demonstrate, the throughput loss due to $K$-SCG feedback protocol is negligible, which indicates that the $K$ SCG feedback protocol is an effective feedback policy.

Figure 4 depicts average interference power at the PBS under $\operatorname{CoS}_{\mathrm{TPIL}}^{K}$ for $K_{N}=N^{0.5}$ when STSB channel gains

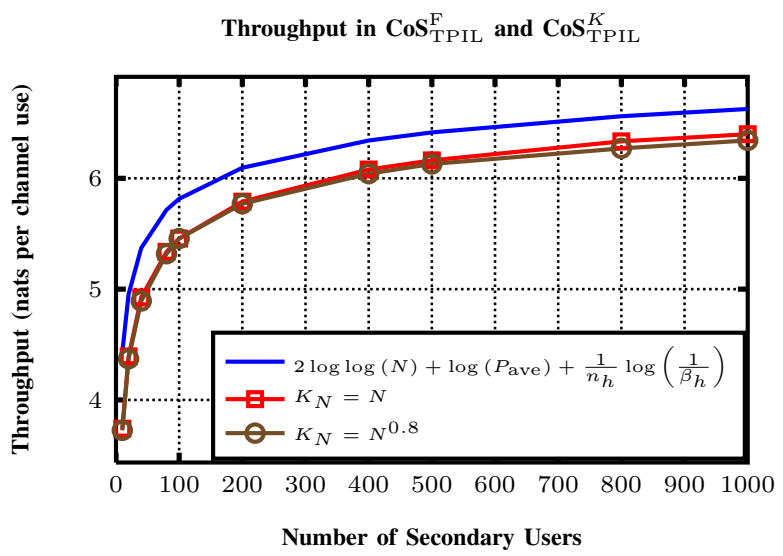

Fig. 3. Secondary network throughput scaling under $\operatorname{CoS}_{\mathrm{TPIL}}^{\mathrm{F}}$ and $\operatorname{CoS} \mathrm{S}_{\mathrm{TPIL}}^{K}$. $P_{\text {ave }}$ and $Q_{\text {ave }}$ are set to $15 \mathrm{~dB}$ and $0 \mathrm{~dB}$, respectively. STSB and STPB channel gains are distributed according to Weibull and Nakagami- $m$ fading models, respectively, with $c=1$ and $m=0.5$.

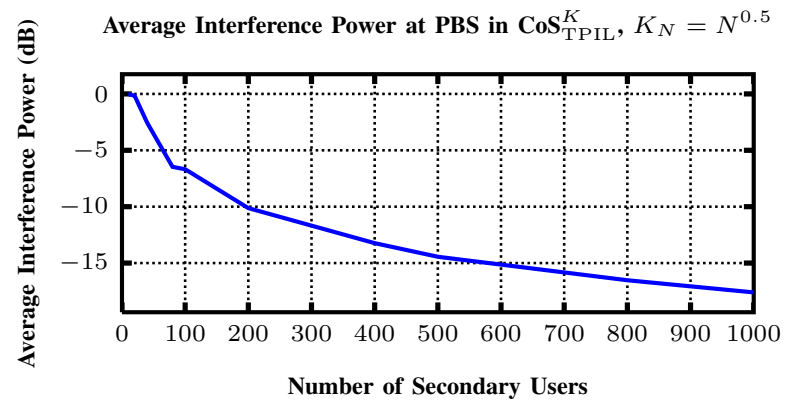

Fig. 4. Average interference power at PBS under $\operatorname{CoS}_{\mathrm{TPIL}}^{K} K_{N}=N^{0.5}$. $P_{\text {ave }}$ and $Q_{\text {ave }}$ are set to $15 \mathrm{~dB}$ and $0 \mathrm{~dB}$, respectively.

are distributed according to the Weibull fading model with $c=1$, and STPB channel gains are Nakagami- $m$ distributed with $m=0.5$. As Fig. 4 shows, the average interference power at the PBS under $\operatorname{CoS}_{\mathrm{TPIL}}^{K}$ converges to zero as the number of SUs becomes large.

\section{CONCLUSION}

In this paper, we have proposed an efficient primarysecondary feedback protocol called $K$-smallest channel gains ( $K$-SCG) feedback control protocol in which the PBS feeds back the $K_{N}$ smallest STPB gains to the SBS. We have examined the effect of $K$-SCG feedback protocol on the throughput scaling behavior for total power and interference limited networks in which transmit powers of SUs are limited by an average total power constraint as well as a constraint 
on the average total interference power that they cause to the PBS. It has been shown that for $K_{N}=N^{\delta}$ with $\delta \in(0,1), K$ SCG feedback protocol is asymptotically optimal. This result implies that the amount of primary-secondary feedback load can be reduced dramatically while the secondary network preserves optimal throughput scaling behavior. It has also been shown that the interference power at the PBS converges to zero almost surely and in mean as $N$ becomes large if $K_{N}$ grows to infinity at a rate $K_{N}=o(N)$.

\section{APPENDIX A}

The asymptotic behavior of $R_{\text {TPIL }}^{\mathrm{F}}(N)$ and $R_{\mathrm{TPIL}}^{K}\left(K_{N}\right)$ depends on the asymptotic behavior of $X_{N}^{\star}\left(\lambda_{N}, \mu_{N}\right)=\max _{1 \leq i \leq N} \frac{h_{i}}{\lambda_{N}+\mu_{N} g_{i}}$ and $X_{K_{N}}^{\star}\left(\lambda_{N}, \mu_{N}\right)=$ $\max _{1 \leq i \leq K_{N}} \frac{h_{\pi(i)}}{\lambda_{N}+\mu_{N} g_{\pi(i)}}$, respectively. Since Lagrange multipliers $\lambda_{N}$ and $\mu_{N}$ change with $N,\left\{\frac{h_{i}}{\lambda_{N}+\mu_{N} g_{i}}\right\}_{i=1}^{N}$ and $\left\{\frac{h_{\pi(i)}}{\lambda_{N}+\mu_{N} g_{\pi(i)}}\right\}_{i=1}^{K_{N}}$ form triangular arrays of random variables which complicates the analysis to some extent. To simplify our analysis, first, we study the asymptotic behavior of $X_{N}^{\star}(\lambda, \mu)$ and $X_{K_{N}}^{\star}(\lambda, \mu)$ for some fixed non-negative real numbers $\lambda$ and $\mu$. Then, we use this result to obtain the asymptotic behavior of $R_{\mathrm{TPIL}}^{\mathrm{F}}(N)$ and $R_{\mathrm{TPIL}}^{K}\left(K_{N}\right)$.

The next four assisting lemmas play a key role in the proof Theorem 1. In the next Lemma, we study the concentration behavior of the extreme order statistic of a sequence of i.i.d. random variables as the number of elements in the sequence grows large. Later, this result will play a central role in deriving the scaling behaviors of $R_{\text {TPIL }}^{\mathrm{F}}(N)$ and $R_{\text {TPIL }}^{K}\left(K_{N}\right)$.

Lemma 2: Let $\left\{Y_{i}\right\}_{i=1}^{N}$ be a sequence of i.i.d. random variables with a common $\mathrm{CDF} F(x)$ such that $F(x)<1$ for $x<\infty$. Let tail behavior of $F(x)$ be characterized by $G(x)$, i.e, $\lim _{x \rightarrow \infty} G(x)(1-F(x))=1$. Also, let $Y_{N}^{\star}=$ $\max _{1 \leq i \leq N} Y_{i}$. Then, for any $\epsilon$ belonging to $(0,1)$, we have

$$
\lim _{N \rightarrow \infty} \operatorname{Pr}\left\{G^{-1}\left(N^{1-\epsilon}\right)<Y_{N}^{\star} \leq G^{-1}\left(N^{1+\epsilon}\right)\right\}=1 .
$$

Proof: Since $\lim _{x \rightarrow \infty} G(x)(1-F(x))=1$, we can express $F_{N}^{\star}(x)$ as $F_{N}^{\star}(x)=\mathrm{e}^{-N \Theta\left(\frac{-1}{G(x)}\right)}$ for $x$ large enough. By using this expression for $F_{N}^{\star}(x)$, we have:

$$
\begin{aligned}
\operatorname{Pr}\left\{Y_{N}^{\star} \leq G^{-1}\left(N^{1+\epsilon}\right)\right\} & =\mathrm{e}^{-N \Theta\left(\frac{1}{N^{1+\epsilon}}\right)} \\
& =1-\Theta\left(\frac{1}{N^{\epsilon}}\right)
\end{aligned}
$$

and

$$
\begin{aligned}
\operatorname{Pr}\left\{Y_{N}^{\star} \leq G^{-1}\left(N^{1-\epsilon}\right)\right\} & =\mathrm{e}^{-N \Theta\left(\frac{1}{N^{1-\epsilon}}\right)} \\
& =\mathrm{e}^{-\Theta\left(N^{\epsilon}\right)}
\end{aligned}
$$

for all $\epsilon \in(0,1)$ and $N$ large enough. Therefore, we have

$$
\operatorname{Pr}\left\{G^{-1}\left(N^{1-\epsilon}\right)<Y_{N}^{\star} \leq G^{-1}\left(N^{1+\epsilon}\right)\right\}=1-\Theta\left(\frac{1}{N^{\epsilon}}\right)
$$

which complets the proof.
Let $\tilde{R}(N, \lambda, \mu)=\mathrm{E}\left[\log \left(X_{N}^{\star}(\lambda, \mu)\right) 1_{\left\{X_{N}^{\star}(\lambda, \mu) \geq 1\right\}}\right]$. Next lemma characterizes the asymptotic behavior of $\tilde{R}(N, \lambda, 0)$, which will be helpful in upper bounding the sum-rate in $\mathrm{CoS}_{\mathrm{TPIL}}^{\mathrm{F}}$.

Lemma 3: For $\lambda>0, \lim _{N \rightarrow \infty} \frac{\tilde{R}(N, \lambda, 0)}{\log \left(\frac{1}{\lambda}\left(\frac{1}{\beta_{h}} \log \left(\alpha_{h} N\right)\right)^{\frac{1}{n_{h}}}\right)}=$ 1.

$$
\text { Proof: Let } \tilde{X}_{N}^{\star}(\lambda, 0)=\frac{\log \left(X_{N}^{\star}(\lambda, 0)\right) 1_{\left\{X_{N}^{\star}(\lambda, 0) \geq 1\right.}}{\log \left(\frac{1}{\lambda}\left(\frac{1}{\beta_{h}} \log \left(\alpha_{h} N\right)\right)^{\frac{1}{n_{h}}}\right)} .
$$

Hence, to show the desired result, it is enough to show $\lim _{N \rightarrow \infty} \mathrm{E}\left[\tilde{X}_{N}^{\star}(\lambda, 0)\right]=1$. Note that $X_{N}^{\star}(\lambda, 0)=\frac{1}{\lambda} h_{N}^{\star}$, where $h_{N}^{\star}=\max _{1 \leq i \leq N} h_{i}$. Recall $F_{h}(x)$ is the probability distribution function common to all $h_{i}, 1 \leq i \leq N$. From Definition 1, the tail behavior of $F_{h}(x)$ is characterized as $G(x)=\frac{x^{-l_{h}}}{\alpha_{h}} \mathrm{e}^{\beta_{h} x^{n_{h}}-H(x)}$. Hence, we can write $G^{-1}(x)=\left(\frac{\frac{1}{\beta_{h}} \log \left(\alpha_{h} x\right)}{1-\frac{l_{h} \log \left(G^{-1}(x)\right)+H\left(G^{-1}(x)\right)}{\beta\left(G^{-1}(x)\right)^{n} h}}\right)^{\frac{1}{n_{h}}}$. Note that $1-$ $\frac{l_{h} \log \left(G^{-1}(x)\right)+H\left(G^{-1}(x)\right)}{\beta_{h}\left(G^{-1}(x)\right)^{n_{h}}}=1-o(1)$. Thus, $G^{-1}(x)=$ $\left(\frac{1}{\beta_{h}} \log \left(\alpha_{h} x\right)\right)^{\frac{1}{n_{h}}}(1+o(1))$. Using Lemma 2, we have

$\operatorname{Pr}\left\{G^{-1}\left(\alpha_{h}^{-\epsilon} N^{1-\epsilon}\right)<h_{N}^{\star} \leq G^{-1}\left(\alpha_{h}^{\epsilon} N^{1+\epsilon}\right)\right\}$

$=\operatorname{Pr}\left\{\frac{(1-\epsilon)^{\frac{1}{n}}(1+o(1))}{\left(\frac{1}{\beta_{h}} \log \left(\alpha_{h} N\right)\right)^{\frac{1}{n_{h}}}}<h_{N}^{\star} \leq \frac{(1+\epsilon)^{\frac{1}{n}}(1+o(1))}{\left(\frac{1}{\beta_{h}} \log \left(\alpha_{h} N\right)\right)^{\frac{1}{n_{h}}}}\right\}$

$=1-\Theta\left(\frac{1}{N^{\epsilon}}\right)$

for $N$ large enough. This final result implies that $\tilde{X}_{N}^{\star}(\lambda, 0) \stackrel{i . p .}{\longrightarrow} 1$ as $N$ goes to infinity, where i.p. stands for in probability. Since convergence in probability does not always imply convergence in mean, we need to show that $\left\{\tilde{X}_{N}^{\star}(\lambda, 0)\right\}_{N=1}^{\infty}$ is uniform integrable. The proof of uniform integrability follows from standard techniques of mathematical analysis, and is omitted due to space limitations.

The next lemma will assist us to upper and lower lower bound the sum-rate in $\operatorname{CoS}_{\mathrm{TPIL}}^{K}$.

Lemma 4: For $\lambda>0, \mu>0$ and $K_{N}$ growing to infinity at a rate $K_{N}=o(N)$, we have $\lim _{N \rightarrow \infty} \frac{\tilde{R}\left(K_{N}, \lambda, \mu\right)}{\log \left(\frac{1}{\lambda}\left(\frac{1}{\beta_{h}} \log \left(\alpha_{h} K_{N}\right)\right)^{\frac{1}{n_{h}}}\right)}=1$.

$$
\text { Proof: Let } \tilde{X}_{K_{N}}^{\star}(\lambda, \mu)=\frac{\log \left(X_{K_{N}}^{\star}\left(\lambda_{h}, \mu\right)\right) 1\left\{X_{K_{N}}^{\star}(\lambda, \mu) \geq 1\right\}}{\log \left(\frac{1}{\lambda_{h}}\left(\frac{1}{\beta_{h}} \log \left(\alpha_{h} K_{N}\right)\right)^{\frac{1}{n_{h}}}\right)} \text {. }
$$

First, we show that $\tilde{X}_{K_{N}}^{\star}(\lambda, \mu) \stackrel{\text { i.p. }}{\longrightarrow} 1$ as $N$ grows large. To this end, we will show that $g_{\pi\left(K_{N}\right)}$ converges to zero in probability. The CDF of the $K_{N}$ th smallest value for the collection of random variables $\left\{g_{i}\right\}_{i=1}^{N}$, which we denote as $F_{g}^{\left(K_{N}\right)}(x)$, is given by

$$
F_{g}^{\left(K_{N}\right)}(x)=\int_{0}^{F_{g}(x)} \frac{x^{K_{N}-1}(1-x)^{N-K_{N}}}{\mathrm{~B}\left(K_{N}, N-K_{N}+1\right)} d x
$$


where $\mathrm{B}(a, b)=\int_{0}^{1} t^{a-1}(1-t)^{b-1} d t$ is the beta function [10], and $F_{g}(x)$ is the CDF common to all $g_{i}, 1 \leq i \leq N$. We define $z_{K_{N}}$ as $z_{K_{N}}=F_{g}\left(g_{\left.\pi_{\left(K_{N}\right)}\right)}\right)$. Using (9), the CDF of $z_{K_{N}}$, which we denote as $F_{z_{K_{N}}}(x)$, is given as

$$
\begin{aligned}
F_{z_{K_{N}}}(x) & =\operatorname{Pr}\left\{z_{K_{N}} \leq x\right\} \\
& \stackrel{(\mathrm{a})}{=} \operatorname{Pr}\left\{g_{K_{N}: N} \leq F_{g}^{-1}(x)\right\} \\
& =\int_{0}^{x} \frac{x^{K_{N}-1}(1-x)^{N-K_{N}}}{\mathrm{~B}\left(K_{N}, N-K_{N}+1\right)} d x,
\end{aligned}
$$

where (a) follows from the fact that $F_{g}(x)$ is invertible and monotone increasing for $x>0$ and $g_{\pi_{\left(K_{N}\right)}}=g_{K_{N}: N}$. Note that the random variable $X$ is said to be Beta distributed with parameters $v$ and $w$ if its $\mathrm{CDF}$ is given by $F_{X}(x)=$ $\int_{0}^{x} \frac{t^{v-1}(1-t)^{w-1}}{\mathrm{~B}(v, w)} d t$. Thus, $z_{K_{N}}$ is indeed a Beta distributed random variable with parameters $K_{N}$ and $N-K_{N}+1$. Using the fact that $z_{K_{N}}$ is Beta distributed, we can upper bound the tail probability $\operatorname{Pr}\left\{g_{\pi\left(K_{N}\right)}>\epsilon\right\}$ of $g_{\pi\left(K_{N}\right)}$ for all $\epsilon>0$ as

$$
\begin{aligned}
\operatorname{Pr}\left\{g_{\pi\left(K_{N}\right)}>\epsilon\right\} & =\operatorname{Pr}\left\{F_{g}\left(g_{\pi\left(K_{N}\right)}\right)>F(\epsilon)\right\} \\
& =\operatorname{Pr}\left\{z_{K_{N}}>F(\epsilon)\right\} \\
& \stackrel{\text { (a) }}{\leq} \frac{\mathrm{E}\left[z_{K_{N}}\right]}{F(\epsilon)} \\
& \stackrel{\text { (b) }}{=} \frac{K_{N}}{F(\epsilon)(N+1)},
\end{aligned}
$$

where (a) follows from the Markov inequality, and (b) follows from the formula $\mathrm{E}\left[z_{K_{N}}\right]=\frac{K_{N}}{N+1}$ for the mean value of Beta distributed random variables [11]. This implies $g_{\pi\left(K_{N}\right)} \stackrel{i . p .}{\longrightarrow} 0$ as $N$ grows large. We will use this convergence property of $g_{\pi\left(K_{N}\right)}$ while we obtain a tight lower bound for $X_{K_{N}}^{\star}(\lambda, \mu)$, hence for $\tilde{X}_{K_{N}}^{\star}(\lambda, \mu)$, below.

We note that the collection of random variables $\left\{h_{\pi(i)}\right\}_{i=1}^{K_{N}}$ are i.i.d. with the common distribution $F_{h}(x)$ because $\boldsymbol{h}$ and $\boldsymbol{g}$ are independent, and our selection criterion depends on $\boldsymbol{g}$. Since $g_{\pi\left(K_{N}\right)}$ is the largest value in $\left\{g_{\pi(i)}\right\}_{i=1}^{K_{N}}$, $X_{K_{N}}^{\star}(\lambda, \mu)$ can be lower bounded as $\max _{1 \leq i \leq K_{N}} \frac{h_{\pi(i)}}{\lambda+\mu g_{\pi\left(K_{N}\right)}} \leq$ $X_{K_{N}}^{\star}(\lambda, \mu)$. Therefore, we can upper and lower bound $X_{K_{N}}^{\star}(\lambda, \mu)$ as $\frac{h_{K_{N}}^{\star}}{\lambda+\mu g_{\pi\left(K_{N}\right)}} \leq X_{K_{N}}^{\star}(\lambda, \mu) \leq \frac{h_{K_{N}}^{\star}}{\lambda}$, where $h_{K_{N}}^{\star}=\max _{1 \leq i \leq K_{N}} h_{\pi(i)}$. From continuous mapping theorem [12], we have $\frac{1}{1+\frac{\mu}{\lambda} g_{\pi\left(K_{N}\right)}} \stackrel{i . p .}{\longrightarrow} 1$ as $N$ grows large, which implies $\frac{\frac{1}{1+\frac{\mu}{\lambda} g_{\pi\left(K_{N}\right)}} \frac{h_{K_{N}}^{\star}}{\lambda}}{\frac{1}{\lambda}\left(\frac{1}{\beta_{h}} \log \left(\alpha_{h} K_{N}\right)\right)^{\frac{1}{n_{h}}}} \stackrel{i . p .}{\longrightarrow} 1$ as $N$ grows large by using Lemma 3. Thus, $\frac{X_{K_{N}}^{\star}(\lambda, \mu)}{\frac{1}{\lambda}\left(\frac{1}{\beta_{h}} \log \left(\alpha_{h} K_{N}\right)\right)^{\frac{1}{n_{h}}}}$ converges to 1 in probability, which, in turn, implies the convergence of $\tilde{X}_{K_{N}}^{\star}(\lambda, \mu)$ to 1 in probability. The proof of uniform integrability is omitted due to space limitations.

In the next lemma, we state an important convergence property for the Lagrange multiplier $\lambda_{N}$ as $N$ grows large. This result will be used to obtain lower and upper bounds for the sum-rate in $\operatorname{CoS}_{\mathrm{TPIL}}^{K}$ and to show the logarithmic effect of $P_{\text {ave }}$ on the secondary network throughput under $\operatorname{CoS}_{\mathrm{TPIL}}^{K}$.
Lemma 5: Let $\lambda_{N}$ be the Lagrange multiplier corresponding to the average total transmit power constraint in $\operatorname{CoS}_{\mathrm{TPIL}}^{K}$. Then, $\lim _{N \rightarrow \infty} \lambda_{N}=\frac{1}{P_{\text {ave }}}$.

Proof: The proof is similar to that of Lemma 6 in [6].

\section{A. Proof of Throughput Scaling in $\operatorname{CoS}_{\mathrm{TPIL}}^{K}$}

We first note that $\tilde{R}\left(K_{N}, \lambda, \mu\right)$ is a decreasing function of $\lambda$ and $\mu$. Thus, for any given $\epsilon>0$, we can find a constant $N_{0}$ large enough such that $R_{\mathrm{TPIL}}^{K}\left(K_{N}\right)$ can be upper and lower bounded as

$$
\tilde{R}\left(K_{N}, \frac{1+\epsilon}{P_{\text {ave }}}, \frac{1}{Q_{\text {ave }}}\right) \leq R_{\mathrm{TPIL}}^{K}\left(K_{N}\right) \leq \tilde{R}\left(K_{N}, \frac{1-\epsilon}{P_{\text {ave }}}, 0\right)
$$

for all $N \geq N_{0}$ since $\lambda_{N}$ converges to $\frac{1}{P_{\text {ave }}}$ by Lemma 5 and $\mu_{N} \leq \frac{1}{Q_{\text {ave }}}$. Using Lemma 4, for any given $\epsilon>0$ and $N$ large enough, $R_{\mathrm{TPIL}}^{K}\left(K_{N}\right)$ can be further upper and lower bounded as (12) which implies $\lim _{N \rightarrow \infty} \frac{R_{\mathrm{TPIL}}^{K}\left(K_{N}\right)}{\log \left(\log \left(K_{N}\right)\right)}=\frac{1}{n_{h}}$.

\section{B. Proof of Throughput Scaling in $\operatorname{CoS}_{\mathrm{TPIL}}^{\mathrm{F}}$}

Consider a secondary network under $\operatorname{CoS}_{\mathrm{TPIL}}^{\mathrm{F}}$ with a total average transmit power constraint $P_{\text {ave }}$, total average interference power constraint $Q_{\text {ave }}$ and $N$ SUs. By removing the average interference power constraint, we obtain a primary MAC network with a total average transmit power constraint $P_{\text {ave. }}$. Hence, the sum-rate in $\operatorname{CoS}_{\mathrm{TPIL}}^{\mathrm{F}}$ can be upper bounded by the sum-rate $R_{\mathrm{TPL}}(N)$ of the primary MAC network with the same total average transmit power constraint, i.e., $R_{\mathrm{TPIL}}^{\mathrm{F}}(N) \leq R_{\mathrm{TPL}}(N)$. In the next lemma, we establish the asymptotic scaling behavior of $R_{\mathrm{TPL}}(N)$, which will also serve as an upper bound for $R_{\mathrm{TPIL}}^{\mathrm{F}}(N)$.

Lemma 6: $R_{\mathrm{TPL}}(N) \quad$ scales according to $\lim _{N \rightarrow \infty} \frac{R_{\mathrm{TPL}}(N)}{\log \log (N)}=\frac{1}{n_{h}}$. Furthermore, for any given $\epsilon>0$, there exists a constant $N_{0}$ large enough such that $R_{\text {TPL }}(N)$ can be upper and lower bounded as (13) for all $N \geq N_{0}$.

Proof: Since the proof is similar to the analysis given above, we skip it to avoid repetitions.

The proof of throughput scaling in $\operatorname{CoS}_{\mathrm{TPIL}}^{\mathrm{F}}$ is completed if we obtain a lower bound for $R_{\mathrm{TPIL}}^{\mathrm{F}}(N)$ that also scales according to $\frac{1}{n_{h}} \log \log (N)$ as $N$ grows large. To this end, we observe that $R_{\mathrm{TPIL}}^{K}\left(K_{N}\right)$ serves as a lower bound for $R_{\mathrm{TPIL}}^{\mathrm{F}}(N)$ since more information is available at the SBS to perform power control and user scheduling under $\operatorname{CoS}_{\mathrm{TPIL}}^{\mathrm{F}}$. Thus, for any given $\epsilon>0,0<\delta<1$ and $N$ large enough, we have (14) which implies $\lim _{N \rightarrow \infty} \frac{R_{\mathrm{TPIL}}^{\mathrm{F}}(N)}{\log (\log (N))}=\frac{1}{n_{h}}$.

\section{APPENDIX B}

Since $\mathcal{I}\left(K_{N}\right)$ is a positive random variable, it is enough to show that $\lim _{N \rightarrow \infty} \mathrm{E}\left[\mathcal{I}\left(K_{N}\right)\right]=0$ for $K_{N}$ increasing to infinity at a rate $K_{N}=o(N)$ as $N$ goes to infinity. We can upper bound $\mathcal{I}\left(K_{N}\right)$ as

$$
\begin{aligned}
\mathcal{I}\left(K_{N}\right) & =g_{i_{K_{N}}^{\star}} P_{i_{K_{N}}^{\star}, K_{N}}^{\star}(\boldsymbol{h}, \boldsymbol{g}) \\
& \leq \frac{g_{K_{N}: N}}{\lambda_{N}},
\end{aligned}
$$


$(1-\epsilon)\left[\log \left(\frac{P_{\text {ave }}}{1+\epsilon}\right)+\frac{1}{n_{h}} \log \left(\frac{1}{\beta_{h}} \log \left(\alpha_{h} K_{N}\right)\right)\right] \leq R_{\mathrm{TPIL}}^{K}\left(K_{N}\right) \leq(1+\epsilon)\left[\log \left(\frac{P_{\text {ave }}}{1-\epsilon}\right)+\frac{1}{n_{h}} \log \left(\frac{1}{\beta_{h}} \log \left(\alpha_{h} K_{N}\right)\right)\right]$

$$
(1-\epsilon)\left[\log \left(\frac{P_{\text {ave }}}{1+\epsilon}\right)+\frac{1}{n_{h}} \log \left(\frac{1}{\beta_{h}} \log \left(\alpha_{h} N\right)\right)\right] \leq R_{\mathrm{TPL}}(N) \leq(1+\epsilon)\left[\log \left(\frac{P_{\text {ave }}}{1-\epsilon}\right)+\frac{1}{n_{h}} \log \left(\frac{1}{\beta_{h}} \log \left(\alpha_{h} N\right)\right)\right]
$$

$$
(1-\epsilon)\left[\log \left(\frac{P_{\text {ave }}}{1+\epsilon}\right)+\frac{1}{n_{h}} \log \left(\frac{1}{\beta_{h}} \log \left(\alpha_{h} N^{\delta}\right)\right)\right] \leq R_{\text {TPIL }}^{\mathrm{F}}(N) \leq(1+\epsilon)\left[\log \left(\frac{P_{\text {ave }}}{1-\epsilon}\right)+\frac{1}{n_{h}} \log \left(\frac{1}{\beta_{h}} \log \left(\alpha_{h} N\right)\right)\right]
$$

where $i_{K_{N}}^{\star}=\pi\left(\arg \max _{1 \leq i \leq K_{N}} \frac{h_{\pi(i)}}{\lambda_{N}+\mu_{N} g_{\pi(i)}}\right)$. Hence, using the fact that $\liminf _{N \rightarrow \infty} \bar{\lambda}_{N}>0$, it is enough to show that $\lim _{N \rightarrow \infty} \mathrm{E}\left[g_{K_{N}: N}\right]=0$. To this end, for any given $\epsilon>0$, we can upper bound $\mathrm{E}\left[g_{K_{N}: N}\right]$ as

$$
\begin{aligned}
\mathrm{E}\left[g_{K_{N}: N}\right] & \\
& =\mathrm{E}\left[g_{K_{N}: N} 1_{\left\{g_{K: N}<\epsilon\right\}}\right]+\mathrm{E}\left[g_{K_{N}: N} 1_{\left\{\epsilon \leq g_{K}: N<N\right\}}\right] \\
& +\sum_{i=1}^{\infty} \mathrm{E}\left[g_{K_{N}: N} 1_{\left\{N^{i} \leq g_{K: N}<N^{i+1}\right\}}\right] \\
& \leq \epsilon+N \operatorname{Pr}\left\{g_{K_{N}: N} \geq \epsilon\right\}+\sum_{i=1}^{\infty} N^{i+1} \operatorname{Pr}\left\{g_{K_{N}: N} \cdot \geq N^{i}\right\}
\end{aligned}
$$

Below, we will show that $\operatorname{Pr}\left\{g_{K_{N}: N} \geq \epsilon\right\}$ and $\operatorname{Pr}\left\{g_{K_{N}: N} \geq N^{i}\right\}$ can be asymptotically bounded as $\operatorname{Pr}\left\{g_{K_{N}: N} \geq \epsilon\right\} \leq \mathrm{e}^{-\Theta(N)}$ and $\operatorname{Pr}\left\{g_{K_{N}: N} \geq N^{i}\right\} \leq$ $\mathrm{e}^{-\Theta\left(N^{i n+1}\right)}$. Assuming for a while that these asymptotic bounds hold, $\mathrm{E}\left[g_{K_{N}: N}\right]$ can be upper bounded as

$$
\begin{aligned}
\mathrm{E}\left[g_{K_{N}: N}\right] & \leq \epsilon+N \mathrm{e}^{-\Theta(N)}+\sum_{i=1}^{\infty} N^{i+1} \mathrm{e}^{-\Theta\left(N^{i n+1}\right)} \\
& \leq \epsilon+N \mathrm{e}^{-\Theta(N)}+N \mathrm{e}^{-N} \sum_{i=1}^{\infty} N^{i} \mathrm{e}^{-\Theta\left(N^{i n}\right)}
\end{aligned}
$$

which implies $\lim \sup _{N \rightarrow \infty} \mathrm{E}\left[g_{K_{N}: N}\right]=0$ and completes the proof.

Now, we derive the aforementioned asymptotic expansions for $\operatorname{Pr}\left\{g_{K_{N}: N} \geq \epsilon\right\}$ and $\operatorname{Pr}\left\{g_{K_{N}: N} \geq N^{i}\right\}$. Note that $\operatorname{Pr}\left\{g_{K_{N}: N} \leq x\right\}=\sum_{i=K_{N}}^{N}\left(\begin{array}{c}N \\ i\end{array}\right) F_{g}^{i}(x)\left(1-F_{g}(x)\right)^{N-i}[10]$. Therefore, for all $x>0$ and $N$ large enough, $\operatorname{Pr}\left\{g_{K_{N}: N} \geq x\right\}$ can be upper bounded as

$$
\begin{aligned}
\operatorname{Pr}\left\{g_{K_{N}: N} \geq x\right\} & =\sum_{i=0}^{K_{N}-1}\left(\begin{array}{c}
N \\
i
\end{array}\right) F_{g}^{i}(x)\left(1-F_{g}(x)\right)^{N-i} \\
& \stackrel{(a)}{\leq} K_{N}\left(\begin{array}{c}
N \\
K_{N}
\end{array}\right)\left(1-F_{g}(x)\right)^{N-K_{N}} \\
& \stackrel{(b)}{\leq} \frac{\sqrt{N} K_{N} 2^{N H_{b}\left(\frac{K_{N}}{N}\right)}}{\sqrt{\pi K_{N}\left(N-K_{N}\right)}}\left(1-F_{g}(x)\right)^{N-K_{N}} \\
& \leq N 2^{N H_{b}\left(\frac{K_{N}}{N}\right)}\left(1-F_{g}(x)\right)^{N-K_{N}},
\end{aligned}
$$

where $(a)$ follows from the fact that $K_{N}<(N+1) F_{g}(x)-1$ for $x>0$ and $N$ large enough, (b) follows from $\left(\begin{array}{c}N \\ K_{N}\end{array}\right) \leq$ $2^{N H_{b}\left(\frac{K_{N}}{N}\right)} \sqrt{\frac{N}{\pi K_{N}\left(N-K_{N}\right)}}$ for $K_{N} \notin\{0, N\}$, where $H_{b}(\cdot)$ is the binary entropy function [13]. Using (15), $\operatorname{Pr}\left\{g_{K_{N}: N} \geq \epsilon\right\}$ can be upper bounded as

$$
\begin{aligned}
\operatorname{Pr}\left\{g_{K_{N}}: N\right. & \geq \epsilon\} \\
& \leq N 2^{N H_{b}\left(\frac{K_{N}}{N}\right)}\left(1-F_{g}(\epsilon)\right)^{N-K_{N}} \\
& =\mathrm{e}^{N\left(\left(1-\frac{K_{N}}{N}\right) \log \left(1-F_{g}(\epsilon)\right)+H_{b}\left(\frac{K_{N}}{N}\right) \log (2)+\frac{\log (N)}{N}\right)} \\
= & \mathrm{e}^{\Theta(-N)}
\end{aligned}
$$

Using similar technique, it is easy to show that $\operatorname{Pr}\left\{g_{K_{N}: N} \geq N^{i}\right\} \leq \mathrm{e}^{-\Theta\left(N^{i n+1}\right)}$.

\section{REFERENCES}

[1] A. Goldsmith, S.A. Jafar, I. Maric and S. Srinivasa, "Breaking spectrum gridlock with cognitive radios: an information theoretic perspective," Proc. IEEE, vol. 97, no. 5, pp. 894-914, May 2009.

[2] A. Ghasemi and E. S. Sousa, "Fundamental limits of spectrum-sharing in fading environments," IEEE Trans. Wireless Commun., vol. 6, no. 2, pp. 649-658, Feb. 2007.

[3] J. M. Peha, "Approaches to spectrum sharing," IEEE Commun. Mag., vol. 43, no. 2, pp. 10-12, Feb. 2005.

[4] T. W. Ban, W. Choi, B. C. Jung, D. K. Sung, "Multi-user diversity in a spectrum sharing system," IEEE Trans. Wireless Commun., vol. 8, no. 1, pp. 102-106, Jan. 2009.

[5] R. Zhang and Y-C. Liang, "Investigation on multiuser diversity in spectrum sharing based cognitive radio networks," IEEE Commun. Letters, vol. 14, no. 2, pp. 133-135, Feb. 2010.

[6] E. Nekouei, H. Inaltekin and S. Dey, "Throughput scaling in cognitive multiple access with average power and interference constraints," IEEE Trans. Signal Process., vol. 60, no. 2, Feb.2012.

[7] A. Tajer and X. Wang, "Multiuser diversity gain in cognitive networks," IEEE/ACM Trans. Netw., vol. 18, no. 6, pp. 1766-1779, Dec. 2010.

[8] H. Wang, J. Lee, S. Kim and D. Hong, "Capacity of secondary users exploiting multispectrum and multiuser diversity in spectrum-sharing environments," IEEE Trans. Veh. Technol., vol. 59, no. 2, pp. 1030-1036, Feb. 2010.

[9] R. Zhang, S. Cui and Y-C. Liang, "On ergodic sum capacity of fading cognitive multiple-access and broadcast channels," IEEE Trans. Inf. Theory, vol. 55, no. 11, pp. 5161-5178, Nov. 2009.

[10] H.A. David and H.N. Nagaraja, Order Statistics, Wiley, New Jersey, 2003.

[11] N.A.J. Hastings and J.B. Peacock, Statistical Distributions, New York: Wiley, 1975.

[12] P. Billingsley, Probability and Measure, New York: John Wiley and Sons, Third edition, 1995.

[13] T.M. Cover and J.A. Thomas, "Elements of Information Theory", New York: Wiley, 2005. 